\title{
Reversible Shape and Plasmon Tuning in Hollow
}

\section{AgAu Nanorods}

Sadegh Yazdi, ${ }^{1}$ Josée R. Daniel, ${ }^{2}$ Nicolas Large, ${ }^{3 \#}$ George C. Schatz, ${ }^{3}$ Denis Boudreau, ${ }^{2}$ and Emilie Ringe $e^{1,4^{*}}$

${ }^{1}$ Department of Materials Science and NanoEngineering, Rice University, Houston, Texas 77005, United States

${ }^{2}$ Center for Optics, Photonics and Lasers (COPL), Department of Chemistry, Laval University, Québec (QC), Canada, G1V 0A6

${ }^{3}$ Department of Chemistry, Northwestern University, Evanston, Illinois 60208, United States

${ }^{4}$ Department of Chemistry, Rice University, Houston, Texas 77005, United States

TOC GRAPHIC

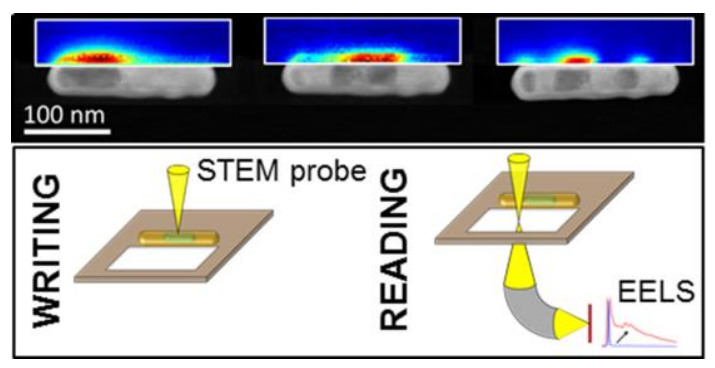

ABSTRACT 
The internal structure of hollow AgAu nanorods created by partial galvanic replacement was manipulated reversibly and its effect on optical properties was mapped with nanometer resolution. Using the electron beam in a scanning transmission electron microscope to create solvated electrons in an encapsulated solution-filled cavity in the nanorods, Ag ions were reduced, reshaping the core of the nanoparticles without affecting the external shape. The resulting changes in plasmon-induced near-field properties were then mapped with electron energy-loss spectroscopy without disturbing the internal structure, and the results are supported by finite-difference time-domain calculations. This reversible shape and near-field control in a hollow nanoparticle actuated by an external stimulus introduces possibilities for applications in reprogrammable sensors, responsive materials, and optical memory units. Moreover, the liquidfilled nanorod cavity offers new opportunities for in situ microscopy of chemical reactions.

\section{KEYWORDS}

Localized surface plasmon resonance (LSPR), Electron energy-loss spectroscopy (EELS), Galvanic replacement, Bimetallic nanorods, Reconfigurable systems, Plasmon near-field

\section{ARTICLE TEXT}

When an external electric field with proper frequency is applied, the conduction electrons of metal nanoparticles (NPs) are driven in a coherent oscillation called localized surface plasmon resonance (LSPR). LSPRs result in wavelength-dependent photon absorption, photon scattering, and local enhancement of the electric field. Absorption and scattering yield strong colors that have been utilized centuries ago in medieval stained glass. Decades ago, the local electric field enhancement was understood to contribute to surface-enhanced spectroscopies such as Raman, ${ }^{1}$ fluorescence, ${ }^{2}$ and absorption. ${ }^{3}$ Since then, the development and application of plasmonics have 
expanded and diversified to include sensors, catalysts, and solar cells, to name a few. ${ }^{4-6}$ This expansion is in part due to the extreme tunability of LSPR properties, such as the position and strength of the absorption, scattering, and near-field enhancement, achievable by changing the particle shape, size, composition, and surrounding dielectric environment. ${ }^{7}$ Interaction with nearby particles further affects the LSPR through interparticle coupling, leading to a significant spectral shift and a larger local field enhancement at the junction between particles. ${ }^{8,9}$

Among the various nanostructures, hollow NPs are of great interest: the additional degree of freedom provided by the shell thickness results in readily tunable optical properties. ${ }^{10}$ Conveniently, such structures can be obtained by simple galvanic replacement in metallic NPs. It has also been shown that changing the concentricity of the inner cavity of hollow particles changes the distribution of their near-field enhancement: nanoshells (i.e., hollow nanospheres), nanoeggs (i.e., non-concentric hollow nanospheres), and nanocups (i.e., truncated hollow nanospheres) are predicted to behave differently, as do infinite cylinders with an offset core. ${ }^{11-13}$ Hollow particles also have the ability to encapsulate another functional material such as dyes and magnetic particles, ${ }^{14}$ making them promising nanostructures for, e.g., diagnostics imaging and photothermal cancer therapy. ${ }^{15}$ Lastly, hollow nanostructures often offer high stability, increased surface area and have potential for economy of material/reduced density. ${ }^{16}$

The tremendous flexibility of hollow nanostructures is, however, currently limited by their static LSPR properties. Dynamic and/or reversible self-assembly techniques have been developed for simple NP shapes, for instance electrons have been injected in plasmonic NPs ${ }^{17}$ while coupled systems have been created by electrochemically regulating a redox surface process in dimers ${ }^{18}$ or using a reversible linker (e.g., DNA $\left.{ }^{19}\right)$. These techniques manipulate both the LSPR energy and near-field by creating hot spots, or regions of high local electric field enhancement, between the 
NPs. The control of these systems is limited by the distance or medium between the NPs such that the pattern of the near-field is only minimally manipulated. A NP that could reversibly and repeatedly be reshaped would offer the advantage of near-field tunability not achievable in coupled systems.

Here, we use the electron beam in a scanning transmission electron microscope (STEM) to manipulate the internal structure of individual hollow $\mathrm{AgAu}$ nanorods in a reversible manner, thus leading to a reversible tuning of the corresponding plasmon-induced near-field distribution. Near-field properties are mapped with nanometer resolution using electron energy-loss spectroscopy (EELS) without disturbing the internal structure, and compared to finite-difference time-domain (FDTD) calculations. This report of the reversible near-field control in a hollow NP actuated by an external stimulus not only introduces possibilities for applications in reprogrammable sensors, responsive materials, and optical multistate memory units, but also provide new means to perform and monitor chemistry in a nanoscopic volume.

The partial galvanic replacement of Ag nanospheres and nanorods by Au in citrate-capped NPs immersed in a gold(III) chloride trihydrate $\left(\mathrm{HAuCl}_{4}\right)$ solution yields partially hollow nanospheres and nanorods, respectively (Figure 1 and Supporting Information, Figure S2). As observed for other structures,${ }^{20}$ continuous reduction of $\mathrm{Au}$ ions on the surface and lateral diffusion of atoms through the particle during the reaction provides a pinhole-free, alloyed metal shell. STEM-high angle annular dark-field (STEM-HAADF) images of drop-cast NPs on $10 \mathrm{~nm}$ thick $\mathrm{Si}_{3} \mathrm{~N}_{4}$ membranes (Figure 1b) show a brighter shell and dimmer core. Since STEMHAADF contrast is roughly proportional to $Z^{1.8}$, where $Z$ is the atomic number, ${ }^{21}$ this indicates that the shell is composed of a higher $Z$ material than the core. The inner material is not, however, solid Ag, as confirmed by cross-sectioning the NPs using focused ion beam (FIB) 
milling. Imaging this cross-section with secondary electrons in a scanning electron microscope (SEM, Figure 1c) shows that the NP cores are hollow. Further, imaging a thin cross-sectional specimen (Supporting Information, Section 5) in a STEM (Figure 1d) confirmed the hollowness of the nanospheres and nanorods.

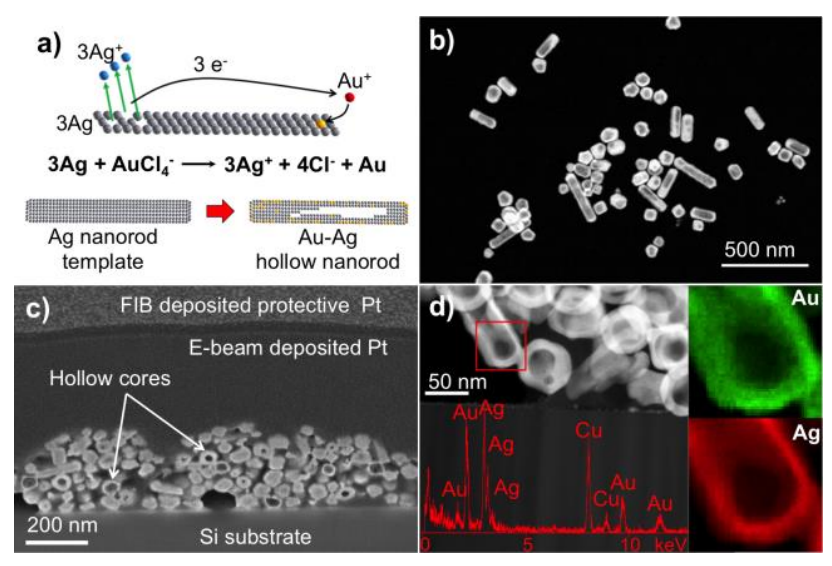

Figure 1. AgAu NPs structure and composition. a) Galvanic replacement process leading to hollow AgAu nanorods. b) Representative STEM-HAADF image of AgAu NPs. c) SEM (secondary electrons) image of a FIB-milled cross-section of a large aggregate of NPs. d) STEMHAADF and EDS spectrum and maps of the FIB-milled cross-section. The Au and Ag maps were generated from the background subtracted L emission lines, and the $\mathrm{Cu}$ in the EDS spectra is from the support $\mathrm{Cu}$ grid.

The hollow cavity is, in fact, filled with an aqueous solution originating from the synthesis medium. While FT-IR measurements in the water absorption region were inconclusive due to trace amounts of water in both precursor and hollow NPs, thermogravimetric analysis shows a weight loss for the hollow particles corresponding to the expected water weight, while no loss was observed for precursor Ag NPs (Supporting Information, Figure S3). Also, core-loss EELS from the $\mathrm{AgAu}$ NPs at the $\mathrm{O} \mathrm{K}$-edge is consistent with the near-edge fine structure of water $^{22}$ and different from the O K-edge of silver oxides ${ }^{23}$ (Supporting Information, Figure S4). This solution 
is apparently sealed inside the particles during galvanic replacement; the shells are thick and appear pinhole-free (Figure 1 and Supporting Information, Figure S2) as also observed by other groups. $^{24}$

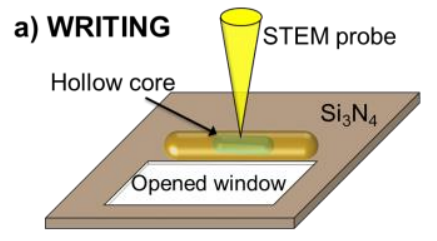

b) READING

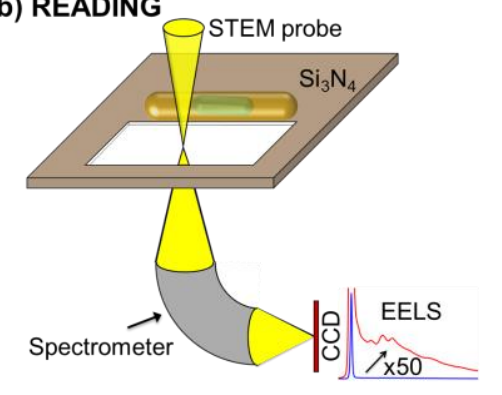

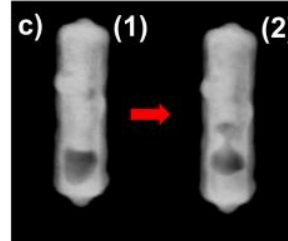

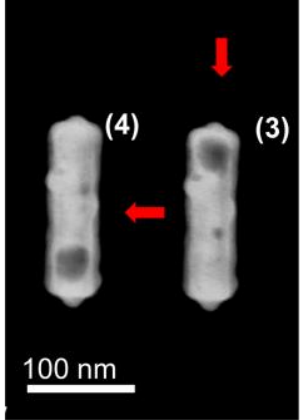

Figure 2. Write/read cycle and reversibility of interior restructuring in partially hollow AgAu nanorods. a) Ag migrates towards the beam when a solution-filled volume is illuminated. b) The void position and its effect on plasmon resonances can be interrogated without restructuring by acquiring electron energy-loss spectra (EELS) from the surroundings of the nanorod. c) Successive reshaping states of the inner structure shows reversibility.

The extent of galvanic replacement in bimetallic systems dictates the composition of the final product. ${ }^{25}$ Here, the replacement was not carried out to completion in order to obtain semihollow internal structures; therefore Ag remains in the final NPs. STEM-energy dispersive X-ray spectroscopy (STEM-EDS) maps in Figure 1 show an Au-rich shell and predominantly Ag core. The Ag:Au atomic ratio increased from 52:48 in the shell to 70:30 over the core, as expected from a surface-initiated galvanic replacement. Bulk Ag:Au atomic ratios of 71:29 and 78:22 ( $1 \%$ error) were obtained by inductively coupled plasma-atomic emission spectroscopy (ICP- 
AES) and SEM-EDS over a large area, respectively (Supporting Information, Table S2). These are in good agreement and further confirm the STEM-EDS results.

Illuminating the solution-filled cavity of the nanorods with a sub-nanometer diameter electron beam $(200 \mathrm{pA}, 80 \mathrm{kV})$ for $3-4$ seconds induces $\mathrm{Ag}$ migration towards the beam, Figure 2 . This is accompanied by motion of the aqueous solution away from the beam and depletion of the Agrich filling in neighboring areas. Once restructured, the particle shape is stable unless the beam is focused on the newly positioned cavity region. Restructuring was observed for most (10 out of 17) of the examined nanorods and can be attributed to the high mobility of ionic Ag and the beam-induced creation of solvated electrons ${ }^{26}$ in the liquid medium inside the void. A comparable mechanism was invoked by Sutter et al. to explain the differences between liquid cell in situ and ex situ galvanic replacement. ${ }^{27}$ The presence of a liquid is in fact critical for this process; no internal restructuring was observed for cut samples (Figure 1). The loss of liquid through an undetected pinhole may explain the lack of restructuring in a minority of the nanorods, however this is likely caused by a more complete galvanic replacement (lower Ag activity); these rods had more of their core hollow than the other rods. Note that Ag mobility can be thermally enhanced, ${ }^{28}$ however, the several hundred degrees Celsius required are much beyond the heating achieved with the electron beam. ${ }^{26,29}$ Therefore, it is unlikely that a thermal process plays a significant role in the current experiment.

The Ag migration process is reproducible and reversible, as indicated by successive reshaping of the internal structure of multiple nanorods with the electron beam (Figure 2c, 4 and Supporting Information Figure S5). Ag ions consistently migrated close to the region illuminated by the focused beam, producing increasingly broad metallic fills at the expense of the other interior regions. No change in the ability of ions to move and reshape the particle has been observed as a 
function of either reshaping cycles or time in the microscope vacuum (up to a day), indicating the reversibility of the process and the stability of the particles, respectively (Supporting Information Figure S5 and Movie S1). This reversibility also indicates that the process is not driven by Ag oxidation, as was observed in liquid cells by Lewis et al., for instance. ${ }^{30}$ Also, no nanorods burst under the beam, and no outer layer structural degradation was observed, consistent with minimal heating and an Ag-ion driven mechanism, where the Au-rich shell is not affected by the electron beam. Note that while the motion of $\mathrm{Ag}$ is repeatable and reversible, the inner structure of the particles before and after shaping/reshaping cycles is never exactly the same because of roughness and presence of $\mathrm{Au}$ atoms in the trapped solution. As seen in the beginning and final states in Figure $2 \mathrm{c}(1,4)$, the void position is nearly identical after a full motion cycle of the void, but the inner void/internal AgAu interface appears to be slightly different. The volume of aqueous solution contained remains constant, ensuring very similar configurations when the void is at either ends, for instance. The data acquisition difficulties associated with the electron-driven internal structure reshaping are discussed in the Supplementary Information, Section 7.

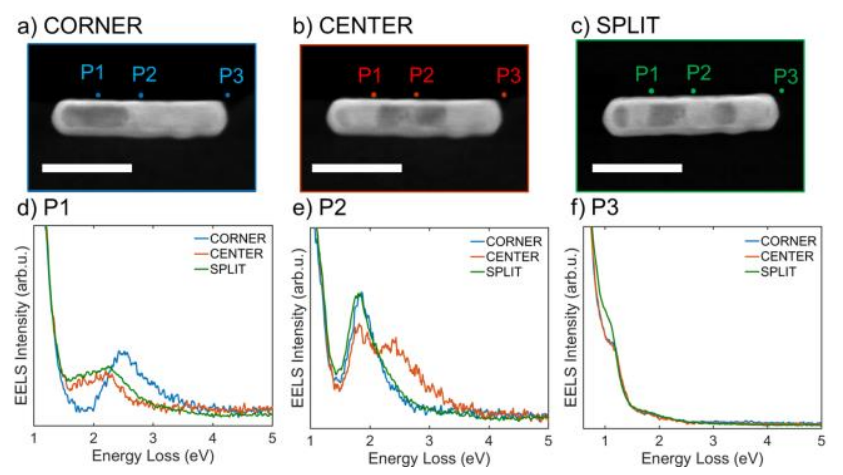

Figure 3. Effect of the internal structure of nanorods on their LSPR response. STEMHAADF images of the same hollow AgAu nanorod after beam-induced internal restructuring, 
with the hollow region a) in a corner, b) at the center, and c) split across the nanorod. $d-f$ ) EELS spectra (normalized by the zero-loss peak intensity) for each internal structure from a single pixel at the positions marked by a circle in panels a-c (P1, P2, and P3). The pixel size is $2.3,2.4$, and $2.2 \mathrm{~nm}$ for the corner, center, and split state, respectively. Scale bars, $100 \mathrm{~nm}$.

The ability to manipulate the near-field spatial distribution of the plasmon modes via internal restructuring was confirmed with EELS (Figures 3-4), a powerful technique providing information about the energy and localization of LSPRs which has already been applied to a variety of shapes. ${ }^{31-33}$ In EELS mapping (also known as spectrum imaging), a nanometer-sized monochromated electron beam is raster-scanned across a sample and an electron energy-loss spectrum is obtained at each point; inelastic scattering processes including contribution from multiple plasmon modes shape the EELS signal in the low-loss region $(<10 \mathrm{eV})$. However, this beam, when traversing the core of the particle, also causes restructuring of hollow nanorods (Figure 2), and the pixel dwell time needed for EELS mapping was too long to insure structural stability. Given that the plasmon near-field properties can be probed outside of the NP, only the external area was mapped, hence completely avoiding reshaping. By opening (milling) a window through the thin membrane with an intense electron beam and acquiring EELS maps only inside this opened window (Figures 2 and S4), the response of nanorods was observed while simultaneously minimizing charging and background effects. After tailoring the inner structure of nanorods with the electron beam (Figure 2), the near-field interaction with an electron beam was recorded using low-loss, monochromated STEM-EELS (Zero-loss peak FWHM $\sim 0.16 \mathrm{eV}$, FEI Titan Themis equipped with a Gatan Quantum ERS electron spectrometer). Figure 3 shows three different void positions for the same nanorod. The void in these internal structure states is located at the corner ("corner" state), at the center ("center" state), and in multiple positions 
along the nanorod ("split" state). Raw EELS spectra obtained by positioning the beam near the particles at one-quarter of their length (P1), their center (P2), and their end tip (P3) show that changing the internal structure of a nanorod leads to changes in its near-field plasmonic response. For instance, much of the electron energy losses, corresponding to plasmon excitations, at P1 (1/4 length) in the corner state appear to be at higher energy than those of the other states, indicating that higher energy plasmons are more strongly excited. Meanwhile, the center state has a larger proportion of high energy losses at P2 (center). Such observations indicate that internal reshaping leads to near-field distribution changes of the plasmon modes.

To generate maps of the plasmon modes, EELS spectrum images were acquired, one for each state (corner, center, and split; full details in the Supporting Information, Section 8). After reshaping the AgAu nanorod from the corner to the center and then to the split state, the corner state was reproduced and an additional EELS spectrum image from this state was acquired to show the reversibility in tuning the LSPRs. Non-negative matrix factorization (NMF), a global fitting tool, was used to fit the spectrum images, as previously reported. ${ }^{34,35}$ Briefly, this approach extracts a user-determined number of spectral components (reported in Figure 4, left panels). For each spectral component, a contribution at each pixel is computed and rendered as a loading map (Figure 4, center panels). The fit spectrum at any given pixel is thus given by a linear combination of the spectral components, where each spectral component is multiplied by its loading at a specific pixel (examples of fit spectra reported in the Supporting Information, Figures S5-S13). NMF fits of the spectrum images for each of the three internal structure states and for the reproduced corner state after the reshaping cycle independently yielded four spectral components corresponding to strong LSPR modes (Figure 4 and Table 1). The lowest energy LSPR peaks at $1.2 \mathrm{eV}$ for all three states; its loss probability intensity is localized at the end of 
the nanorod, representing the primitive longitudinal dipolar LSPR. There is no correlation between the HAADF intensity and the EELS loss probability (black and red line in the rightmost graphs of Figure 4, respectively) suggesting that the near-field for this mode does not depend significantly on the void position, in agreement with FDTD calculations (Supporting Information, Section 9, and Figure S16). The loss probability distribution of this longitudinal LSPR is in excellent agreement with previously reported EELS studies on filled plasmonic nanorods. ${ }^{36}$ The LSPR at $1.8 \mathrm{eV}$ is also present for all void positions and its loss probability spatial distribution is insensitive to the void position; the near-field pattern suggests this resonance is either a higher order $(l=2$, quadrupole) longitudinal mode or a transverse mode; FDTD calculations (vide infra) indicate that it is the latter.

Table 1. LSP mode assignment in EELS measurements, FDTD and EELS-FDTD calculations.

\begin{tabular}{lccc}
\hline \hline LSP mode & $\begin{array}{c}\text { EELS } \\
\text { (experimental) }\end{array}$ & $\begin{array}{c}\text { FDTD } \\
\text { (theory) }\end{array}$ & $\begin{array}{c}\text { EELS-FDTD } \\
\text { (theory) }\end{array}$ \\
\hline Antibonding transverse dipole & $2.5 \mathrm{eV}$ & $2.38 \mathrm{eV}$ & $2.37 \mathrm{eV}$ \\
Bonding quadrupole & $2.1 \mathrm{eV}$ & $2.32 \mathrm{eV}$ & $2.28 \mathrm{eV}$ \\
Bonding transverse dipole & $1.8 \mathrm{eV}$ & $2.15 \mathrm{eV}$ & $2.10 \mathrm{eV}$ \\
Bonding longitudinal dipole & $1.2 \mathrm{eV}$ & $1.30 \mathrm{eV}$ & $1.27 \mathrm{eV}$ \\
\hline \hline
\end{tabular}

More interestingly, the two higher energy LSPRs are strongly affected by the inhomogeneous spatial distribution of polarizable electrons inside the nanorod, due to the void, as well as plasmon hybridization between the void and the nanorod modes (details in Supporting Information, Figure S18). Such coupling provides a rich range of modes, previously shown theoretically and experimentally in concentric spherical gold nanoshells ${ }^{10,37}$ and theoretically in nonconcentric structures. ${ }^{11,12,38}$ Here, the resonance around $2.1 \mathrm{eV}$ has a high loss probability near the inner void/metal interface, located at the center-left for the corner state (Figure 4a), and 
at one-quarter and three-quarters of the nanorod length for the center and split states (Figure 4b,c). This mode is attributed to a bonding quadrupolar resonance. The highest energy mode $(2.5 \mathrm{eV})$ has loss probability peaking adjacent to the void area, as shown by the nearly perfect anticorrelation of the loss probability and the internal HAADF trace signal (right part of Figure 4). This mode is attributed to an antibonding combination of the transverse primitive modes of the void and of the nanorod (Supporting Information, Figure S18).

a) CORNER

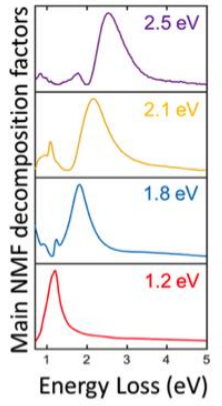

b) CENTER

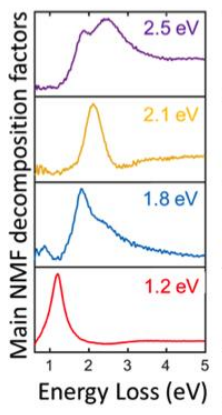

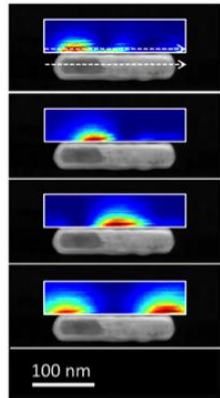
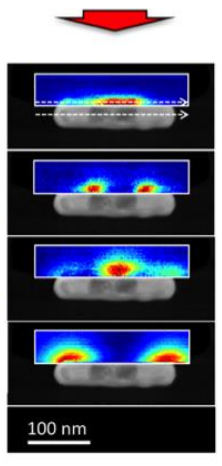
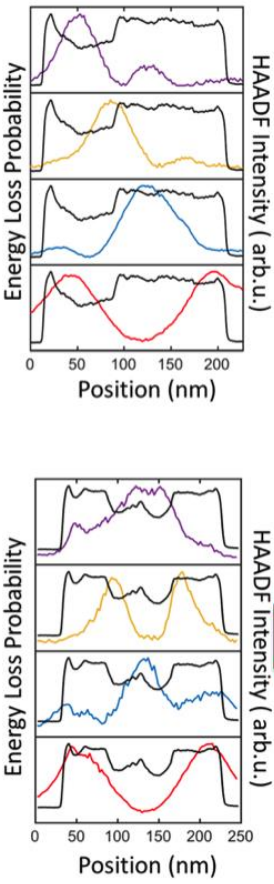

d) CORNER
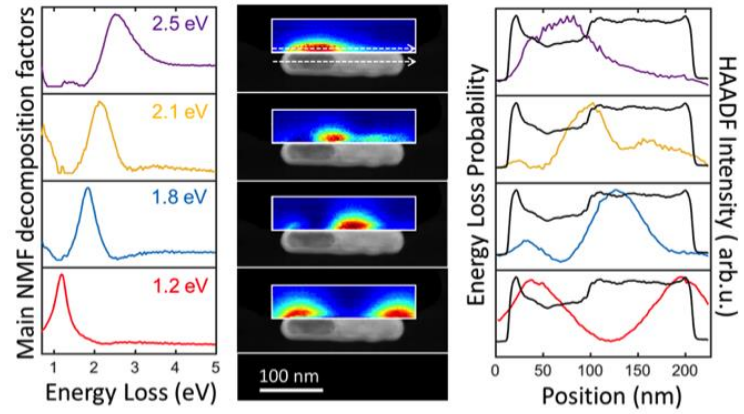

c) SPLIT

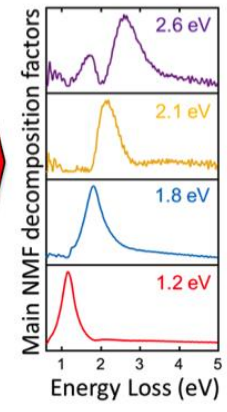

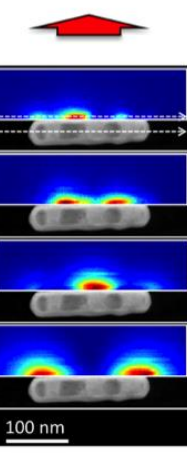

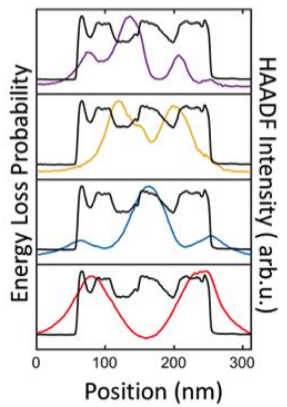

Figure 4. EELS spectra and maps of the three void positions. Plasmon resonances and the relationship between energy electron-loss probability and internal structure in a partially hollow AgAu nanorod for the a) corner state before any reshaping cycle, b) center state, c) split state and d) corner state after a reshaping cycle. NMF decomposition factors and their associated loss probability maps are shown on the left and center parts of each panel, respectively. The crosssection of the spatial distribution of each plasmon mode is superimposed onto the HAADF scattering intensity (right part of the panels) to highlight the correlation between HAADF (void 
position) and EELS (plasmon excitation distribution). White dashed arrows on the EELS maps indicate the position at which the energy loss probability profile and HAADF intensity profile were obtained.

To provide deeper insight into the mode analysis, the commercial package Lumerical FDTD Solutions $^{39}$ was used to solve Maxwell's equations, along with a recently developed ${ }^{40}$ EELSFDTD implementation proven to be successful for complex nanostructures. ${ }^{41}$ Briefly, an electric dipole is placed at different positions along the electron beam trajectory (here taken as the $z$ axis). The electric field induced by this electric dipole in presence of the nanorod is then calculated along the $z$-axis for each dipole position. The loss probability is then given by ${ }^{40}$

$$
\Gamma_{\mathrm{EELS}}(\omega)=\frac{e^{2}}{\pi \hbar \omega^{2}} \iint_{-\infty}^{\infty} \cos \left[\frac{\omega\left(z-z^{\prime}\right)}{v}\right] \times \operatorname{Im}\left[\frac{E_{z}^{\text {ind }}(z, \omega)}{p\left(z^{\prime}, \omega\right)}\right] \mathrm{d} z \mathrm{~d} z^{\prime}
$$

where $p\left(z^{\prime}, \omega\right)$ is the electric dipole placed at $z^{\prime}$ and $E_{z}^{\text {ind }}(z, \omega)$ is the $z$-component of the induced electric field calculated at $z$. The electrons are considered traveling with a velocity $v=c / 2$ (a kinetic energy of $80 \mathrm{keV}$ ). Parameters used include a FDTD domain of $750 \times 750 \times 1500 \mathrm{~nm}$, a simulation time of $50 \mathrm{fs}$, a conformal mesh of $2 \mathrm{~nm}$ to discretize the structure (mesh accuracy set to 4), and a dipole step of $\mathrm{d} z^{\prime}=2 \mathrm{~nm}$. The dielectric permittivity of gold from Johnson and Christy was used for the metallic shell, ${ }^{42}$ while constant refractive indices of 1.33 and 1.00 were used for the water void and surrounding vacuum medium, respectively.

The modeled geometry and dimensions are based on STEM-HAADF images of the nanorod (Figure 3a-c), and can be described as a cylinder with semi-spherical ends, with a length $L_{\mathrm{Au}}=195 \mathrm{~nm}$ and a radial diameter $a_{\mathrm{Au}}=47 \mathrm{~nm}$. The radially concentric water void is also cylindrical with semi-spherical ends with $L_{\text {water }}=80 \mathrm{~nm}$ and $a_{\text {water }}=35 \mathrm{~nm}$. Its relative longitudinal 
position with respect to the center of the gold nanorod is given by $\Delta$.

a)

(1)

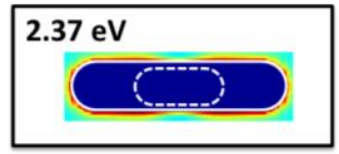

b)

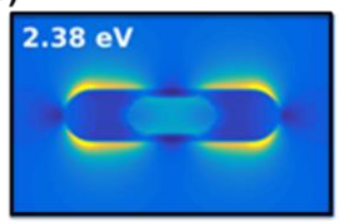

c)

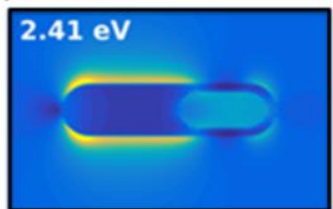

d)

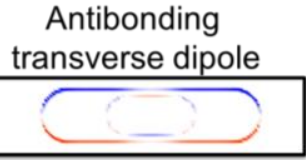

(2)
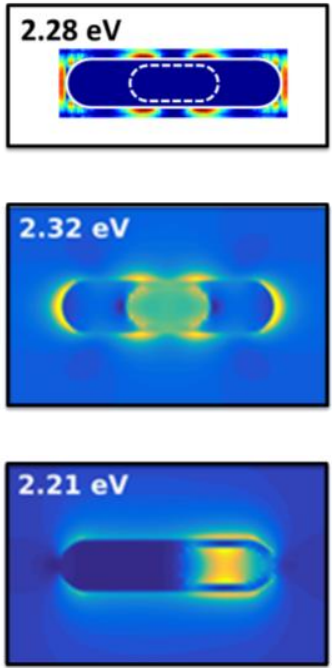

Bonding quadrupole

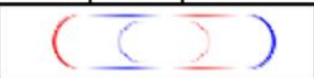

(3)
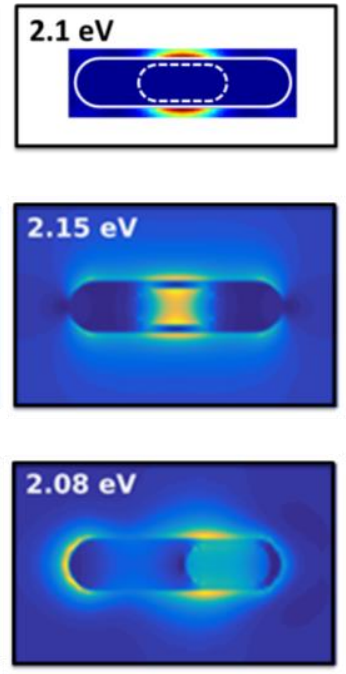

Bonding transverse dipole
(4)
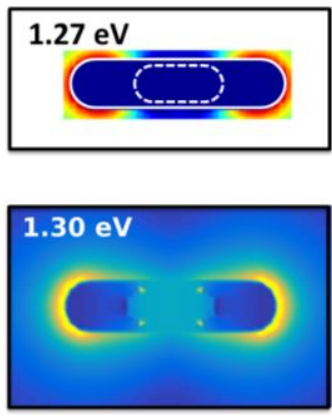

\section{$1.37 \mathrm{eV}$}

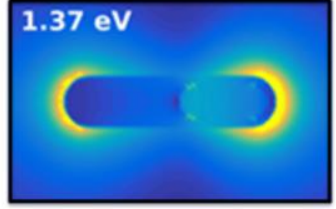

Bonding longitudinal dipole

Figure 5. FDTD and EELS-FDTD numerical results. a) NMF decomposition of the calculated loss probability ( $\Gamma_{\text {EELS }}$, eq. 1$)$ maps calculated using the EELS-FDTD method for the center state nanorod (i.e., $\Delta=0 \mathrm{~nm}) . \mathrm{b}, \mathrm{c})$ Near-field $\left(\left|\mathbf{E} / \mathbf{E}_{0}\right|^{2}\right)$ spatial distribution calculated at each LSPR, for $\Delta=0 \mathrm{~nm}(\mathrm{~b})$ and $\Delta=49 \mathrm{~nm}$ (c). The LSPR energy is indicated in each panel. d) Calculated surface charge density distribution (red: positive charge, blue: negative charge) for each of the four LSP modes obtained in the FDTD calculations from panel $b$.

The calculated EELS spectra (Supporting Information, Figure S17) are in good agreement with the experimental EELS measurements. NMF decomposition of the calculated EELS spectrum image in the center-state case (Figure 5a) reveals the presence of various plasmon modes between 1.27 and $2.37 \mathrm{eV}$, in agreement with standard optical FDTD calculations (Figure 5b,c and Supporting Information, Figure S16). The NMF-extracted EELS-FDTD loss probability maps (Figure 5a) reveal the nature of different plasmon modes and can be directly compared 
with the experimental results (Figure 4b). The FDTD results (near-field distributions, Figure $5 \mathrm{~b}, \mathrm{c}$ ) also agree very well with the mode distribution, despite the difference in excitation (light vs. electron). The LSP mode energies and nature obtained experimentally, along with those calculated with EELS-FDTD and FDTD are reported in Table 1. The modes at 2.1 and $2.37 \mathrm{eV}$ in EELS-FDTD and 2.15, $2.38 \mathrm{eV}$ in FDTD appear to be the bonding and antibonding combinations of the transverse dipoles, respectively (Figure 5a,b(1,3) and Supporting Information, Figure S16b). These two modes, although spectrally shifted from the experimental values, correspond to the modes experimentally observed at 1.8 and $2.5 \mathrm{eV}$, respectively. A quadrupolar mode can be seen at $2.28 \mathrm{eV}(2.1 \mathrm{eV}$ experimentally), also in agreement with optical FDTD calculations (Figures $5 \mathrm{~b}(2)$ and Supporting Information, Figure S14a). The mode at $1.27 \mathrm{eV}$ (Figure 5a(4)) aligns well with the $1.30 \mathrm{eV}$ LSP mode of the optical FDTD calculations (Figures 5b(4) and Supporting Information, Figure S14a), and the $1.2 \mathrm{eV}$ mode observed experimentally. The nature of the modes is further supported by calculated surface charge density distributions (Figure 5d), where positive and negative charges at the surface of the metal are represented in red and blue, respectively. Interestingly, the antibonding transverse dipole $(2.38 \mathrm{eV}$, Figure $5 \mathrm{~b}(1))$ can be observed in optical FDTD calculations, although it is supposed to be a dark mode, due to the slight quadrupolar component coming from the void ( $c f$. Figure $5 \mathrm{~d}(1)$ and Supporting Information, Figure S18a). This additional quadrupolar contribution gives rise to a symmetry breaking, thus inducing a net dipole moment.

The spectral differences between the calculated and measured LSPRs provide crucial insights into the plasmon hybridization in these hollow nanorods. Indeed, the transverse bonding and antibonding dipoles exhibit an energy splitting of $0.7 \mathrm{eV}$ experimentally $(2.5$ and $1.8 \mathrm{eV})$ as compared to $0.27 \mathrm{eV}$ from the EELS-FDTD calculations (2.37 and $2.1 \mathrm{eV})$. This larger splitting, 
i.e., the red- and blue-shift of the antibonding and bonding dipoles, respectively, indicates that the experimental void/nanorod hybridization of the transverse modes is much stronger than in the modeled nanostructure. This suggests that the experimental void/nanorod interface may be thinner than the structure used in FDTD calculations. It can also be noticed from the FDTD results (Figure 5 and Supporting Information, Figure S16) that the longitudinal quadrupolar mode is very close in energy to the transverse bonding and antibonding dipoles. Consequently, small variations in the plasmon hybridization (i.e., small variations in the relative void and nanorod positions) can result in mode cross-overs and energy level re-organization.

In summary, we demonstrated the controllable, reversible, and dynamic tuning of the plasmon near-field properties of hollow AgAu nanorods by using an electron beam to actuate chemical reactions in a nanoscopic trapped aqueous solution. The Ag ions in solution are reduced by solvated electrons, and the internal reshaping process is fully reversible. Further, mapping the nanorod LSPRs with nanometer spatial resolution by STEM-EELS revealed a strong structural dependence and interaction between the void and nanorod plasmons. Plasmon hybridization theory and state-of-the-art FDTD and EELS-FDTD calculations revealed the bonding and antibonding hybridization of the plasmon resonances. The restructuring strategy presented in this work provides tools to study and engineer the plasmonic near-field properties of single supported nanostructures and opens new possibilities to study chemical reactions in nanoscale volumes.

\section{ASSOCIATED CONTENT}

\section{Supporting Information}

The Supporting Information is available free of charge on the ACS Publications website. 
Details of NP synthesis, NP size and shape distribution, results of composition analysis by ICPAES and EDS, thermogravimetric and core-loss EELS analysis, details of cross-sectional TEM specimen preparation, additional details on acquisition and analysis of STEM, EDS, and STEMEELS spectrum images, additional theory information on FDTD calculations, plasmon hybridization, and symmetry dependence of the EELS-FDTD spectra (PDF).

Movie showing 38 consecutive internal structure states, created from 38 consecutive write/image cycles (AVI).

\section{AUTHOR INFORMATION}

\section{Corresponding Author}

*Emilie Ringe, er12@rice.edu. Phone: (773) 348-2582

\section{Author Contributions}

S. Y. and E. R. designed and performed the electron microscopy experiments and data analysis. J. R. D. and D. B. performed synthesis and bulk characterization (TGA, ICP, SEM-EDS), N. L. and G. C. S. performed the FDTD and EELS-FDTD calculations and the theoretical analysis. All authors contributed to writing the paper.

\section{Funding Sources}

N. L. and G. C. S. were supported by NSF grant CHE-1414466. Computing resources were provided by the Quest high performance computing facility at Northwestern University, which is jointly supported by the Office of the Provost, the Office for Research, and Northwestern University Information Technology. D. B. and J. R. D. acknowledge funding from the Natural Sciences and Engineering Research Council of Canada, the Canadian Foundation for Innovation, Fonds de la Recherche du Québec - Nature et Technologies, and U. Laval's "Fonds ArthurLabrie pour la Qualité de l'air" and "Fonds Paul-Antoine Giguère". 
Notes: The authors declare no competing financial interest.; \# Current affiliation: Department of Physics \& Astronomy, The University of Texas at San Antonio, San Antonio, Texas

\section{ACKNOWLEDGMENT}

J. R. D. and D. B. would like to thank Jérémie Asselin, Philippe Legros, and Alexandre Grégoire for insightful discussions, and Stéphan Gagnon for XRD measurements at COPL.

\section{REFERENCES}

(1) Stiles, P. L.; Dieringer, J. A.; Shah, N. C.; Van Duyne, R. P. Annu. Rev. Anal. Chem. 2008, 1, 601-626.

(2) Lakowicz, J. R. Anal. Biochem. 2005, 337, 171-194.

(3) Link, S.; El-Sayed, M. A. J. Phys. Chem. B 1999, 103, 4212-4217.

(4) Brongersma, M. L.; Halas, N. J.; Nordlander, P. Nat. Nanotechnol. 2015, 10, 25-34.

(5) Haes, A. J.; Van Duyne, R. P. Anal. Bioanal. Chem. 2004, 379, 920-930.

(6) Thomann, I.; Pinaud, B. A.; Chen, Z.; Clemens, B. M.; Jaramillo, T. F.; Brongersma, M. L. Nano Lett. 2011, 11, 3440-3446.

(7) Kelly, K. L.; Coronado, E.; Zhao, L. L.; Schatz, G. C. J. Phys. Chem. B 2003, 107, 668677.

(8) Romero, I.; Aizpurua, J.; Bryant, G. W.; García De Abajo, F. J. Opt. Express 2006, 14, 9988.

(9) Hao, E.; Schatz, G. C. J. Chem. Phys. 2004, 120, 357.

(10) Prodan, E.; Radloff, C.; Halas, N. J.; Nordlander, P. Science 2003, 302, 419-422.

(11) Knight, M. W.; Halas, N. J. New J. Phys. 2008, 10, 105006.

(12) Wu, Y.; Nordlander, P. J. Chem. Phys. 2006, 125, 124708.

(13) Zhang, J.; Fu, Y.; Mei, Y.; Jiang, F.; Lakowicz, J. R. Anal. Chem. 2010, 82, 4464-4471. 
(14) Huang, C.; Jiang, J.; Muangphat, C.; Sun, X.; Hao, Y. Nanoscale Res. Lett. 2010, 6, 1-5.

(15) Coughlin, A. J.; Ananta, J. S.; Deng, N.; Larina, I. V.; Decuzzi, P.; West, J. L. Small 2014, $10,556-565$.

(16) Zhang, X.; Zhang, G.; Zhang, B.; Su, Z. Langmuir 2013, 29, 6722-6727.

(17) Mulvaney, P.; Pérez-Juste, J.; Giersig, M.; Liz-Marzán, L. M.; Pecharromán, C. Plasmonics 2006, 1, 61-66.

(18) Byers, C. P.; Hoener, B. S.; Chang, W.-S.; Yorulmaz, M.; Link, S.; Landes, C. F. J. Phys. Chem. B 2014, 118, 14047-14055.

(19) Jin, R.; Wu, G.; Li, Z.; Mirkin, C. A.; Schatz, G. C. J. Am. Chem. Soc. 2003, 125, 16431654.

(20) Skrabalak, S. E.; Chen, J.; Sun, Y.; Lu, X.; Au, L.; Cobley, C. M.; Xia, Y. Acc. Chem. Res. 2008, 41, 1587-1595.

(21) Pennycook, S. J.; Nellist, P. D. Scanning Transmission Electron Microscopy; Pennycook, S. J.; Nellist, P. D., Eds.; Springer New York: New York, NY, 2011.

(22) Aronova, M. A.; Sousa, A. A.; Leapman, R. D. Micron 2011, 42, 252-256.

(23) Boronin, A. .; Koscheev, S. .; Zhidomirov, G. . J. Electron Spectros. Relat. Phenomena 1998, 96, 43-51.

(24) Sun, Y.; Xia, Y. Nano Lett. 2003, 3, 1569-1572.

(25) Zhang, H.; Jin, M.; Wang, J.; Li, W.; Camargo, P. H. C.; Kim, M. J.; Yang, D.; Xie, Z.; Xia, Y. J. Am. Chem. Soc. 2011, 133, 6078-6089.

(26) Egerton, R. F.; Li, P.; Malac, M. Micron 2004, 35, 399-409.

(27) Sutter, E.; Jungjohann, K.; Bliznakov, S.; Courty, A.; Maisonhaute, E.; Tenney, S.; Sutter, P. Nat. Commun. 2014, 5, 4946.

(28) Mallard, W. C.; Gardner, A. B.; Bass, R. F.; Slifkin, L. M. Phys. Rev. 1963, 129. 
(29) Yuk, J. M.; Park, J.; Ercius, P.; Kim, K.; Hellebusch, D. J.; Crommie, M. F.; Lee, J. Y.; Zettl, A.; Alivisatos, A. P. Science (80-. ). 2012, 336, 61-64.

(30) Lewis, E. A.; Slater, T. J. A.; Prestat, E.; Macedo, A.; O’Brien, P.; Camargo, P. H. C.; Haigh, S. J. Nanoscale 2014, 6, 13598-13605.

(31) Genç, A.; Patarroyo, J.; Sancho-Parramon, J.; Arenal, R.; Duchamp, M.; Gonzalez, E. E.; Henrard, L.; Bastús, N. G.; Dunin-Borkowski, R. E.; Puntes, V. F.; Arbiol, J. ACS Photonics 2016, 3, 770-779.

(32) Nicoletti, O.; de la Peña, F.; Leary, R. K.; Holland, D. J.; Ducati, C.; Midgley, P. A. Nature 2013, 502, 80-84.

(33) Koh, A. L.; Bao, K.; Khan, I.; Smith, W. E.; Kothleitner, G.; Nordlander, P.; Maier, S. A.; McComb, D. W. ACS Nano 2009, 3, 3015-3022.

(34) Leary, R. K.; Kumar, A.; Straney, P. J.; Collins, S. M.; Yazdi, S.; Dunin-Borkowski, R. E.; Midgley, P. A.; Millstone, J. E.; Ringe, E. J. Phys. Chem. C 2016, acs.jpcc.6b02103.

(35) Ringe, E.; DeSantis, C. J.; Collins, S. M.; Duchamp, M.; Dunin-Borkowski, R. E.; Skrabalak, S. E.; Midgley, P. A. Sci. Rep. 2015, 5, 17431.

(36) Nicoletti, O.; Wubs, M.; Mortensen, N. A.; Sigle, W.; van Aken, P. A.; Midgley, P. A. Opt. Express 2011, 19, 15371.

(37) Prodan, E.; Nordlander, P. J. Chem. Phys. 2004, 120, 5444.

(38) Moradi, A. Opt. Commun. 2009, 282, 3368-3370.

(39) http://www.lumerical.com/tcad-products/fdtd/. Lumerical Solutions, Inc.

(40) Cao, Y.; Manjavacas, A.; Large, N.; Nordlander, P. ACS Photonics 2015, 2, 369-375.

(41) Zhang, M.; Large, N.; Koh, A. L.; Cao, Y.; Manjavacas, A.; Sinclair, R.; Nordlander, P.; Wang, S. X. ACS Nano 2015, 9, 9331-9339.

(42) Johnson, P. B.; Christy, R. W. Phys. Rev. B 1972, 6, 4370-439. 